\title{
Placental mesenchymal stem cells of fetal and maternal origins demonstrate different therapeutic potentials
}

\author{
Yongzhao Zhu' ${ }^{1,2}$, Yinxue Yang ${ }^{1}$, Yaolin Zhang ${ }^{1,4}$, Guiliang Hao ${ }^{1,4}$, Ting Liu', Libin Wang ${ }^{1,3}$, Tingting Yang ${ }^{1}$,
} Qiong Wang ${ }^{1}$, Guangyi Zhang ${ }^{1}$, Jun Wei ${ }^{1,3^{*}}$ and Yukui Li $i^{1,3^{*}}$

\begin{abstract}
Introduction: Therapeutic potentials of mesenchymal stem cells (MSCs) from different sources have been evaluated in pre-clinical and clinical settings. Although MSCs from different sources share MSC-specific characteristics and functions, inconsistent or controversial results of pre-clinical and clinical applications of such cells are frequently reported. This may be partially due to the fact that MSCs isolated from different origins may differentially express some functions not typical for MSCs, and hence have different therapeutic potentials. The aim of this study is to investigate the differences in human placental MSCs (P-MSCs) of fetal and maternal origins in the aspects of clinical importance.
\end{abstract}

Methods: P-MSCs of fetal and maternal origins isolated from normal term placentas were characterized for their typical phenotype as well as their expression of receptors and growth factors of clinic interests. P-MSCs that preferentially express hepatocyte growth factor (HGF) and CD200 were evaluated for their therapeutic potentials in models of angiogenesis and allogeneic skin transplantation, in comparison with their HGF and CD200 negative partners.

Results: Although all P-MSCs express typical MSC phenotype, fetal but not maternal P-MSCs express high levels of CD200 and HGF. Compared with HGF and CD200 negative P-MSCs, HGF and CD200 positive cells demonstrated significantly high potentials in promoting angiogenesis in vitro and increasing immunosuppressive function in vivo. These therapeutic potentials were at least in part due to their differences in HGF and CD200 expression, respectively.

Conclusions: We conclude that MSC origins may have significant impact on the therapeutic potentials of such cells, and should be taken into consideration in clinical applications.

\section{Introduction}

Mesenchymal stem cells (MSCs) are considered one of the most promising cell types for therapeutic applications and are, hence, most intensively evaluated in preclinical and clinical settings. The characteristics that demonstrate MSC's high therapeutic potentials include the potential for multiple lineage differentiation [1], the ability of secreting growth factors that can promote cell

\footnotetext{
* Correspondence: dreamcell@live.cn; yukuili@hotmail.com

'Institute of Stem Cell Research, General Hospital of Ningxia Medical University, Yinchuan 750004, China

${ }^{3}$ Key Laboratory of Fertility Preservation and Maintenance, Ministry of Education, Ningxia Medical University, Yinchuan 750004, China

Full list of author information is available at the end of the article
}

growth and tissue repair [2]; the function of immunomodulation and immunosuppression [3]; and the limited expression of major histocompatibility complex (MHC) II and co-stimulating molecules which allow MSCs to be used across MHC barriers [4]. Equally noteworthy, not all these characteristics are equally expressed in MSCs of different origins, or in MSCs prepared under different conditions. For instance, CD200, a cell surface molecule mediating an immunosuppressant signal, is expressed in significant levels in some bone marrow-derived MSCs but marginal levels in others, and almost undetectable in MSCs of umbilical cord blood origin [5]. Also, matrix metalloproteinases (MMPs), the molecules that mediate multiple cell-cell interactions and functions of MSCs,

\section{Ciomed Central}


are expressed by MSCs in high levels under inflammation conditions but not under normal physical conditions [6]. Such differences may have significant impact on the outcome of clinical treatments when the MSCs are used for cell therapies. For example, hepatocyte growth factor (HGF) is expressed by some MSC cultures [7]. Standal et al. [8] reported that HGF decreased bone morphogenetic protein (BMP)-induced osteoblast activity and, hence, increased bone loss, in multiple myeloma (MM) bones, and $\mathrm{Xu}$ et al. [9] reported that bone marrow-derived MSCs (BM-MSCs) promoted MM cell growth, protected MM cells from drug-induced apoptosis, and shortened the life span of MM-bearing mice, while Li et al. [10] demonstrated that placenta-derived MSC-like cells prevented bone loss, stimulated bone formation and suppressed growth of $\mathrm{MM}$ in mouse bone. Similar inconsistencies of the therapeutic effects of MSCs from different origins or different preparations on tumor growth have been well documented [11]. These studies together support a notation that MSCs of different origins or prepared under different conditions may express different functions and have different therapeutic potentials.

Of the different sources of MSCs so far investigated, human term placentas have drawn increased interest in recent years $[12,13]$, due mainly to their non-invasive donor procurement and large MSCs supply, in addition to their sharing the basic properties with BM-MSCs [14-17]. A human placenta contains MSCs of both fetal and maternal origin. We [18] and others [13,19,20] have previously isolated and characterized fetal and maternal MSCs from human placentas. Compared with placental MSCs (P-MSCs) of maternal origins, fetal MSCs have demonstrated stronger immunomodulatory function and higher osteogenic differentiation potential $[13,20]$. However, the defined properties of these cells relevant to therapeutic applications are still poorly understood. In the present report, we tested the differences between PMSCs of fetal and maternal origins in the aspects of immunosuppressant molecule expression, growth factor secretion, and functions in stimulating angiogenesis and modulating immunosuppression. The results suggest that MSC origins may have significant impact on the therapeutic potentials of such cells, and should be taken into consideration in clinical applications.

\section{Materials and methods}

Animals

C57BL/6 and Vr:CD1(ICR) mice were obtained from the Surgical Experiment Center of Ningxia General Hospital Affiliated Ningxia Medical University. All animal studies were performed with a protocol approved by the committee of animal care and use at the Ningxia Medical University.

\section{P-MSCs cell isolation and culture}

Human full-term placentas were obtained from healthy mothers at the time of routine elective caesarean section in the Affiliated Hospital of Ningxia Medical University. Informed consent was obtained from each mother prior to delivery. Human placental tissues were collected with a protocol approved by the Ethics Committee for the Conduct of Human Research at Ningxia Medical University. The Human Research Ethics Committee at Ningxia Medical University approved this study. Fetal and maternal P-MSCs were isolated and genetic origin verified as described previously [18]. The cells were cultured in Dulbecco's Modified Eagle Medium (DMEM) supplemented with $10 \%$ fetal bovine serum (FBS), $2 \mathrm{mM}$ L-glutamine and $50 \mu \mathrm{g} / \mathrm{ml}$ gentamycin (Invitrogen, Carlsbad, CA, USA). This medium is referred to as P-MSC medium. All cultures were maintained at $37^{\circ} \mathrm{C}$ in a humidified incubator with $5 \% \mathrm{CO}_{2}$. At about $90 \%$ confluence, the cells were passaged after detachment with TrypLE ${ }^{\mathrm{mm}}$ Express (Invitrogen). All the studies were performed with five passages of the established P-MSCs cultures.

\section{Flow cytometry}

P-MSCs were harvested by TrypLE ${ }^{\mathrm{mix}}$ Express treatment and analyzed by flow cytometry with a FACS Calibur flow cytometer (BD Biosciences, San Diego, CA, USA). All monoclonal antibodies used for flow cytometry were obtained from BD Pharmingen (Franklin Lakes, NJ, USA) and prepared in PBS. Cells $\left(1 \times 10^{6}\right)$ in $100 \mu$ l were incubated with $10 \mu \mathrm{l}$ of one of the following antibodies for 20 minutes at room temperature: IgG1-PE, CD34-PE, CD73-PE, IgG2a-FITC, CD14-FITC, CD45-FITC, CD90FITC, CD105-FITC, CD200-PE or HLA-DR-FITC. After washing, cells were resuspended in $500 \mu \mathrm{L}$ PBS and phenotyped by flow cytometry analysis.

\section{RNA isolation and qRT-PCR}

Cultured fetal and maternal P-MSCs in passage 5 were washed with PBS and total RNA was isolated with Trizol Reagent (Invitrogen). cDNA was elaborated from RNA using Revert Aid First Strand cDNA Synthesis Kit (Thermo Fisher Scientific, San Diego, CA, USA). Quantitative PCR (qPCR) was carried out using the DyNAmo* Capillary SYBR* Green 2-Step qRT-PCR Kit (Thermo Fisher Scientific). Thermal cycling conditions were $95^{\circ} \mathrm{C}$ for 10 minutes followed by 40 cycles of $15 \mathrm{~s}$ at $95^{\circ} \mathrm{C}$, followed by 1 minute at $60^{\circ} \mathrm{C}$. We obtained melt data by 30 minutes at $60^{\circ} \mathrm{C}$. PCR primer sets for CD200, HGF and GAPDH were: CD200: forward 5' - AATACCTTTG GTTTTGGGAAGATCT-3', reverse 5'-GGTGGTCTTC AGAGAATTTGTAGTGA-3'; HGF: forward 5'-ATTGC CCTATTTCTCGTTGTG-3', reverse 5'-GCATTTCTC ATCTCCTCTTCC-3'; GAPDH; forward 5'-AACATCA 
TCCCTGCTTCCAC-3', reverse 5'-GACCACCTGGTC CTCAGTGT-3'.

\section{ELISA}

Equal numbers of fetal and maternal P-MSCs in passage 5 were seeded into $35 \mathrm{~mm}$-Petri dishes, respectively, and cultured in normal medium (as described above) with or

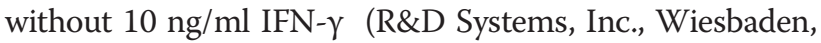
Germany) or $1 \mu \mathrm{M}$ poly (I:C) (Invivogen, San Diego, USA) (final concentration) for $72 \mathrm{~h}$. At the end of cell culture, cell numbers were re-counted and supernatants of each culture were collected for ELISA analysis. All ELISA kits were obtained from BOSTER (Wuhan, China). ELISA analysis was performed according to the manufacturer's protocol. The concentration of each cytokine secreted by P-MSCs is normalized with the cell number at the end of experiments and expressed as $\mathrm{pg} / \mathrm{ml} / 10^{5}$ cells.

\section{Preparation of P-MSC-conditioned medium}

Maternal and fetal P-MSCs in passage 5 were seeded at $1 \times 10^{6}$ cells $/ 100 \mathrm{~mm}$ plate with $10 \mathrm{ml}$ P-MSCs medium and cultured for 48 hours. After this cultivation period, medium from each culture was collected and clarified by centrifugation at $1,000 \mathrm{rpm}$ for 10 minutes at $4^{\circ} \mathrm{C}$. These media were referred to as maternal and fetal P-MSCconditioned medium, respectively.

\section{In vitro angiogenesis assay}

Growth factor-reduced Matrigel (BD Biosciences) was thawed at $4^{\circ} \mathrm{C}$ overnight and spread evenly over 48 -well plates pre-chilled at $4^{\circ} \mathrm{C}$. The plates were incubated for 30 minutes at $37^{\circ} \mathrm{C}$ to allow gel formation. Human umbilical vein endothelial cells (HUVECs) (BIOLEAF, Shanghai, China) were cultured in DMEM with $10 \%$ FBS, $50 \mathrm{U} / \mathrm{ml}$ penicillin and $50 \mathrm{ug} / \mathrm{mL}$ streptomycin (Invitrogen). Cells that reached $80 \%$ confluency were collected by trypsinization and centrifugation. Single cell suspensions were prepared in P-MSCs medium, P-MSCs medium plus $20 \mathrm{ng} / \mathrm{ml}$ recombinant human growth factor (rhHGF) (R\&D System), maternal P-MSC-conditioned medium, fetal P-MSC-conditioned medium, and fetal P-MSCconditioned medium plus $100 \mathrm{ng} / \mathrm{ml}$ anti-human HGF antibody (R\&D System). A total of $3.5 \times 10^{4}$ cells in $500 \mu \mathrm{l}$ of each medium were evenly seeded over a Matrigel-coated well of 48-well plates. After incubation for 12 hours, the cultures were imaged under a microscope and the numbers of capillary tube structures were counted from nine randomly chosen fields for each culture condition.

\section{Skin transplantation}

Vr:CD1(ICR) mice at postnatal Day 3 were used as skin donors, and C57BL/6 mice at age 8 to 10 weeks of age were used as recipients. The donor mice were sacrificed with $\mathrm{CO}_{2}$, and then skin grafts of $1.2 \mathrm{~cm}$ in diameter were prepared using a surgical puncher. The recipient mice were anesthetized with chloral hydrate (Sangon Biotech, Shanghai, China), and a round incision of $1.2 \mathrm{~cm}$ in diameter was made on the dorsal side of each recipient mouse. After the removal of full-thickness skin from the incision sites of the recipient mice, donor skin grafts were transplanted into the sites, and dressed with Vaseline dressings and Band-Aids. At the time of the skin transplantation, the recipient mice were injected (tail i.v.), respectively, with $200 \mu \mathrm{l}$ PBS, $1 \times 10^{5}$ maternal P-MSCs in $200 \mu \mathrm{l} \mathrm{PBS}, 1 \times 10^{5}$ fetal P-MSCs in $200 \mu \mathrm{l}$ PBS and $1 \times 10^{5}$ CD200 blocked fetal P-MSCs. The CD200 of fetal P-MSCs was blocked by using mouse anti-human PE-conjugated CD200 antibody according to the method derived from Mika Pietila et al. [5]. Briefly, fetal P-MSCs were incubated with anti-human CD200 antibody (BD Biosciences) at $20 \mu \mathrm{l}$ antibody $/ 1 \times 10^{6}$ cells in $100 \mu \mathrm{l}$ PBS for 30 minutes and then washed twice with PBS and suspended in PBS. Grafts were inspected daily from the third day of transplantation to establish time of rejection.

\section{Statistical analysis}

All statistical analyses were performed using SPSS statistical analysis software (SPSS Inc., Chicago, IL, USA). Results were expressed as mean \pm SD for illustration. The statistical significance in differences among treatments was determined using one-way ANOVA with a post-hoc least square difference (LSD) test. A $P$-value of less than 0.05 was considered significant.

\section{Results}

Fetal, but not maternal, P-MSCs preferentially express CD200

The P-MSCs of fetal and maternal origins were isolated and characterized as previously described [18]. For matched comparison, the cells were maintained under the same conditions and exposed to the same treatments throughout the experiments, and the results were compared in pairs of individual placenta donors. Both fetal and maternal P-MSCs express typical MSC morphology and phenotype, they all share the similar spindle-like shape (Figure 1A), as well as being positive for CD73, CD90 and CD105, and negative for CD14, CD45, CD34 and MHC class II receptor HLA-DR (Figure 1B). Since CD200 has been shown to mediate the immunosuppressive function to multiple myeloid cell types, especially to dendritic cells and macrophages [21,22], we tested the expression of this molecule in P-MSCs. As shown in Figure 1B, CD200 positive cells count for about $70 \%$ of the fetal P-MSCs population, while being barely detectable in maternal P-MSCs. To confirm this finding, we further isolated P-MSCs from three random donors, and analyzed CD200 expression by qPCR 


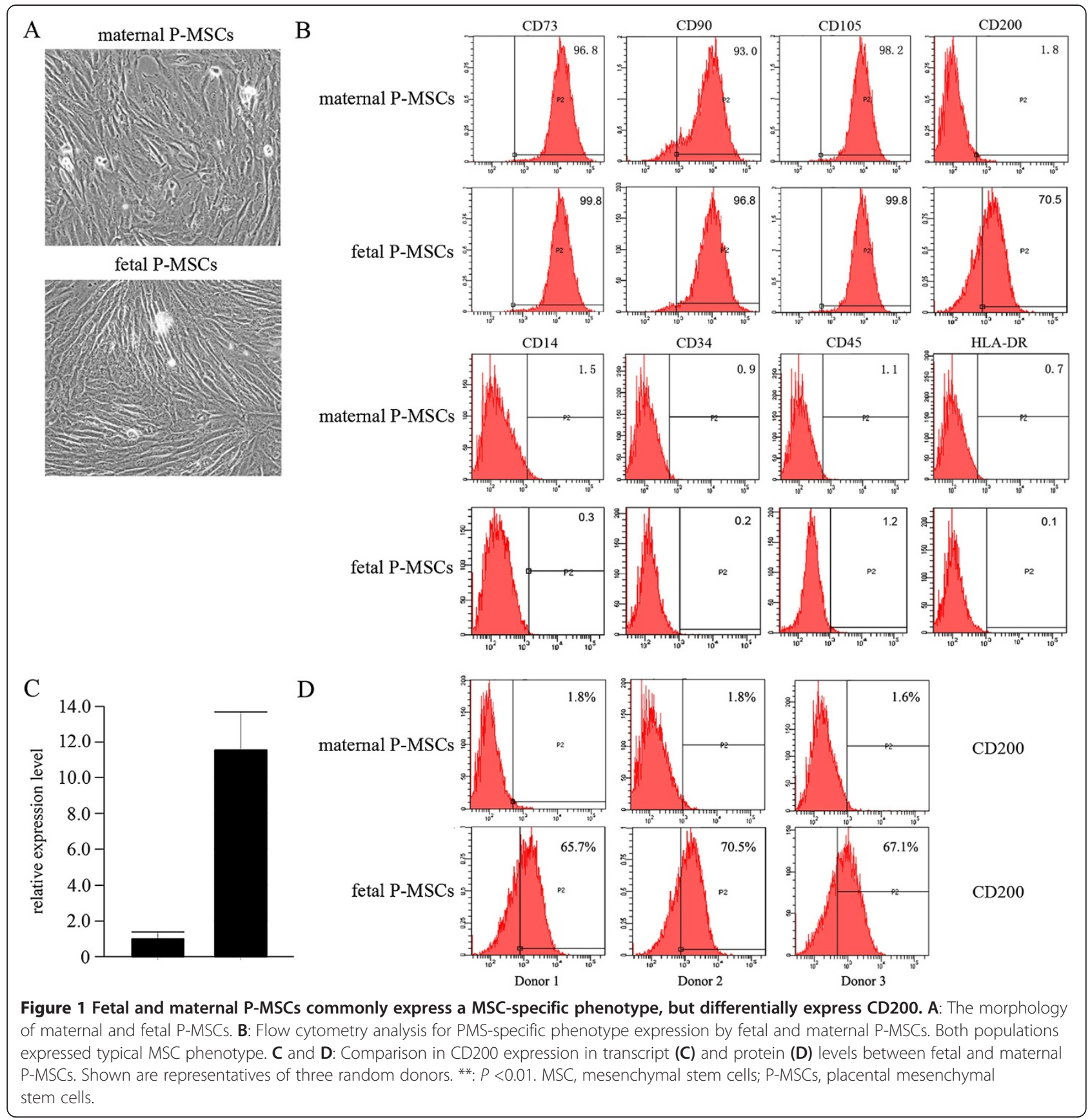

and flow cytometry. As shown in Figure 1C, CD200 transcripts in fetal P-MSCs from the three donors averaged more than 10 times that in maternal P-MSCs (Figure 1C). This finding was further confirmed in protein levels. Flow cytometry demonstrated that $\mathrm{CD}_{200^{+}}$cells were constantly identified in fetal P-MSCs but not in maternal P-MSCs from all three random donors. As CD200 mediates multiple lines of immunosuppressive signals, this result may suggest higher potential of fetal P-MSCs for immunosuppressant treatments.
Fetal P-MSCs express significantly higher levels of HGF than do maternal P-MSCs

HGF is an important growth factor that not only promotes cell growth, morphogenesis and tissue regeneration [23], but also exerts a regulatory role in inducing tolerogenic dendritic cells and regulatory $\mathrm{T}$ cells [24]. We examined HGF along with interleukins 6 (IL-6), 8 (IL-8), 10 (IL-10) and TNF- $\alpha$ expressed in P-MSCs of fetal and maternal origins in cell number-controlled settings (see Materials and methods). Surprisingly, while 
the cytokines were expressed in comparable levels in both cell populations, fetal P-MSCs expressed a much higher level of HGF, about 100 times as much as expressed by maternal P-MSCs do (Figure 2A). We further verified this finding by $\mathrm{qPCR}$ for transcript expression of HGF. As shown in Figure 2B, a significant difference in HGF transcript expression comparable with protein secretion was observed. This result suggests that a superior benefit may be expected for therapeutic applications of fetal P-MSCs over P-MSCs of maternal origin.

\section{Fetal and maternal P-MSCs responded to} immunostimulation with discrete cytokine pathways It has been previously reported that interferon gamma (INF- $\gamma$ ) and Toll like receptor-3 ligand poly (I:C) can induce activation and enhance therapeutic potential of MSCs $[3,25]$. We sought next to elucidate how the P-MSCs of fetal and maternal origin respond to these immune stimuli. Although both cell types responded to the stimuli significantly in the aspect of cytokine induction (Figure 3), they showed discrete cytokine response patterns. Upon INF- $\gamma$ stimulation, fetal P-MSCs significantly increased IL- 6 secretion, and decreased to a lesser but statistically significant extent the secretion of IL-8, IL-10 and HGF; in comparison, in maternal P-MSCs, INF- $\gamma$ induced significant increases in IL-6, IL-10 and HGF (Figure 3A,B). Considering the immunomodulatory and anti-inflammation properties of IL-10 and HGF, this observation may suggest that INF- $\gamma$-priming may be more preferable for maternal P-MSCs than fetal P-MSCs when immunomodulation applications are considered for these cells.

Markedly different from INF- $\gamma$, Poly (I:C) stimulation resulted in a significantly increased secretion of IL-8 in maternal P-MSCs but not fetal P-MSCs, and a significantly increased secretion of HGF in fetal but not maternal P-MSCs. (Figure 3C,D). Whether the different responses are due to differentiated expression of TLR-3 in these two cell populations is unknown and need to be further explored.

\section{Fetal P-MSCs stimulated angiogenesis of HUVECs in vitro} HGF is well known for its function in stimulating angiogenesis of endothelial cells. Having observed that the P-MSCs of fetal origin expressed a much higher level of HGF in both mRNA and protein than P-MSCs of maternal origin (Figure 2), we next tested if this difference would translate into differences in their ability to stimulate angiogenesis. As shown in Figure 4, the human umbilical vein-derived endothelial cells (HUVECs) grew into a limited number of tube structures when cultured in the absence of HGF. When human recombinant HGF was added to the culture, significantly more tube structures were observed. In comparison, when HUVECs were cultured in fetal P-MSCs-conditioned medium, tube formation was enhanced to the level similar to HGF-treated cultures. This enhancement in angiogenesis was not observed when HUVECs were cultured in maternal P-MSCs-conditioned medium, and was inhibited by the addition of anti-HGF antibodies (Figure 4A,B). These results demonstrated that, compared with maternal P-MSCs, fetal P-MSCs expressed an increased ability to stimulate in vitro angiogenesis, and it is suggestive, though not conclusive, that this function is dependent on HGF secretion.

\section{Fetal P-MSCs prolonged skin allograft survival through CD200 expression}

MSCs are known to have immunosuppressive functions. CD200 is a negative regulator of a number of immune cells, predominantly cells of myeloid origin. Since P-MSCs of fetal origin expressed much higher CD200 than P-MSCs of maternal origin, we reasoned the CD200-
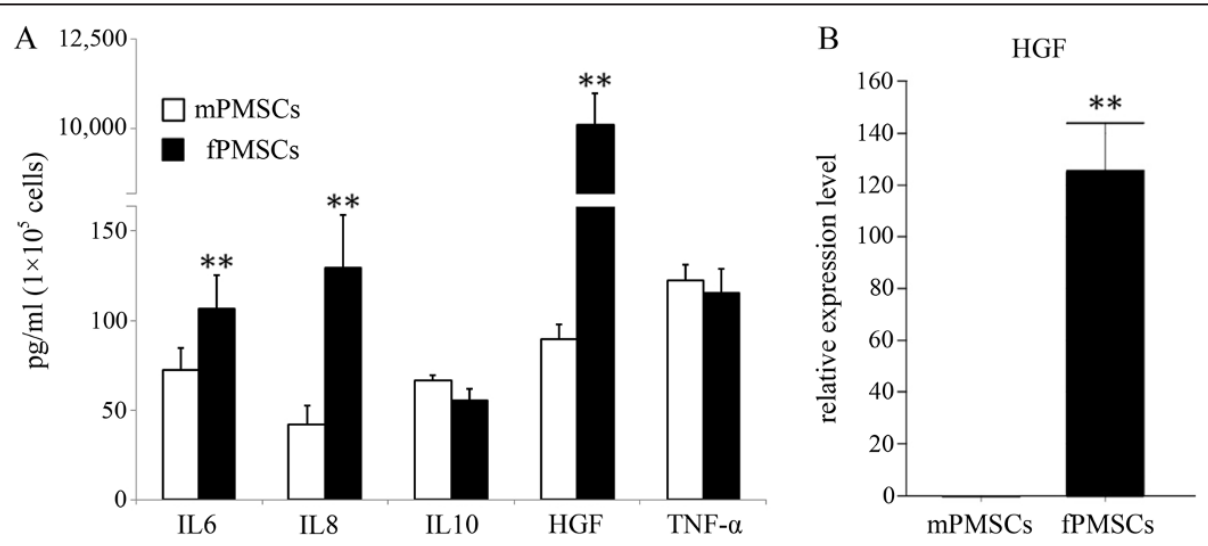

Figure 2 Differential cytokine expression by fetal and maternal P-MSCs. A: P-MSC cells from fetal and maternal P-MSCS were seeded in equal numbers and re-counted at the end of culture. Cytokine levels in cell culture supernatants were quantified by ELISA and normalized by cell numbers at the end of the study. B: qPCR quantification for HGF expression by fetal and maternal P-MSCs. $n=3 . * *$ : $P<0.01$. HGF, hepatocyte growth factor; P-MSCs, placental mesenchymal stem cells. 

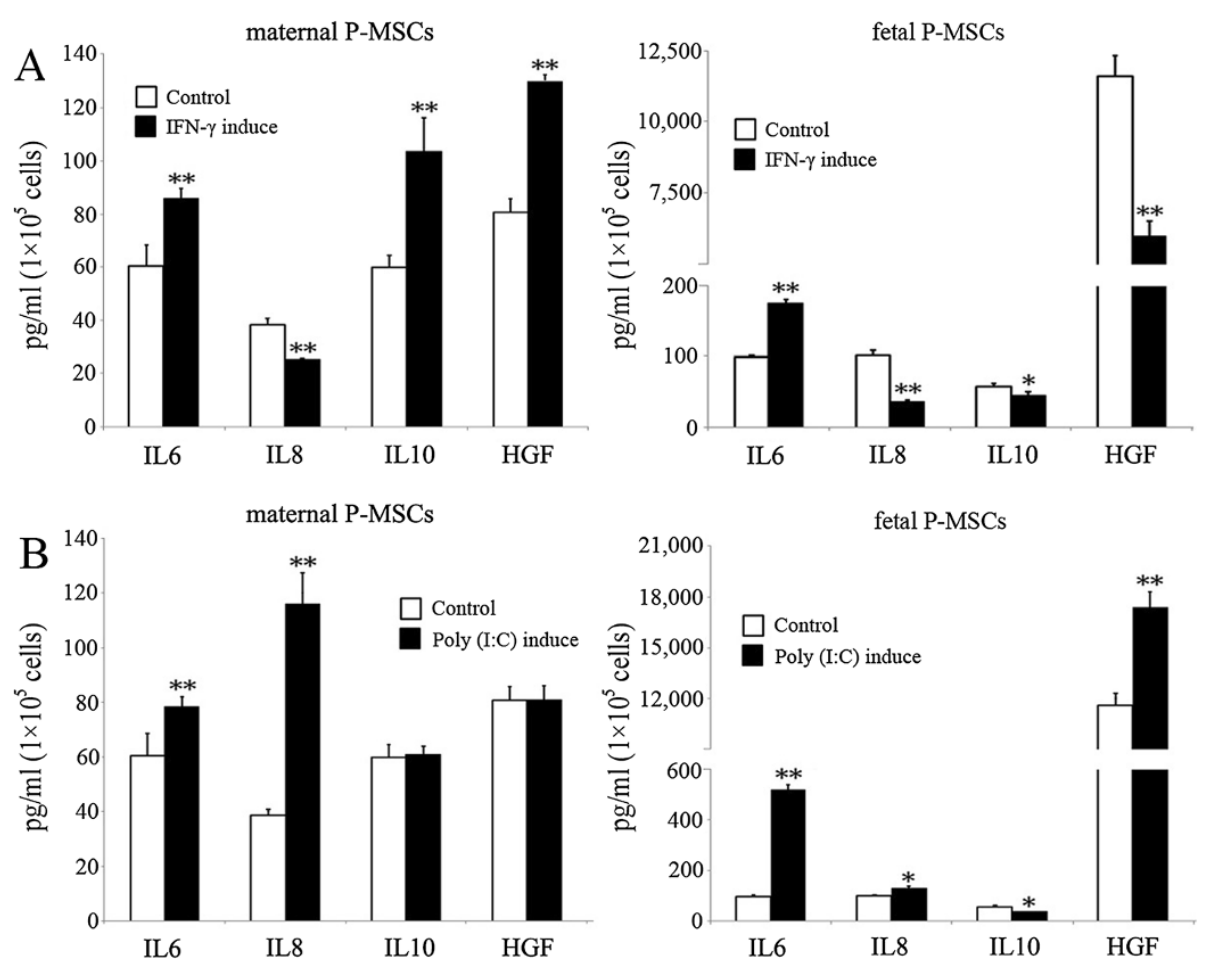

Figure 3 Cytokine expression by fetal and maternal P-MSCs in response to IFN- $\gamma$ and Poly (I:C) stimulations. Equal numbers of cells from fetal and maternal P-MSCs were seeded and stimulated with IFN-ץ (A) and Poly (I:C) (B). Cytokine levels in cell culture supernatants were quantified by ELISA and normalized by cell numbers at the end of the study. $n=3$. ${ }^{*}: P<0.05,{ }^{* *}: P<0.01$. IFN- $\gamma$, interferon gamma; P-MSCs, placental mesenchymal stem cells.

expressing cells should have higher immunosuppressive functions. This was tested in a mouse skin allograft model. As shown in Figure 5, without immunosuppressant, skin allografts were rejected within six days. When the recipient mice received an IV injection of fetal P-MSCs, the allografts survived to 10 days on average. When anti-CD200 antibodies were injected into the CD200-expressing celltreated mice, the graft survival time was reduced to an average of eight days, similar to the survival time in mice treated with CD200-negative cells. These results indicated that fetal P-MSCs prolonged allograft survival, partially but not totally, due to CD200-mediated immunosuppression. Maternal P-MSCs (CD200 negative MSCs) could also increase allograft survival, but to a significantly lower extent than did fetal P-MSCs (CD200 positive MSCs).

\section{Discussion}

MSCs are one of the most promising types of stem cells for recent clinical applications. One existing problem frequently reported with this cell type is the inconsistent or controversial results from pre-clinical and clinical applications, possible reasons, among others, being the different impacts from the sources of the cells, culture systems used and the ratio of different sub-groups with different properties/functions of cells in a given heterogeneous population. In the present study, we started to address this problem by studying paired MSC populations of fetal and maternal origin from the same tissues (individual placentas) and under tightly controlled conditions for their difference in the aspects of clinical relevance. These settings allowed us to define the impact of developmental sources of MSC populations to the cell properties/functions. In this report we have demonstrated that fetal but not maternal P-MSCs express high levels of CD200 and HGF, and respond differently to immunostimulations in terms of cytokine secretion. Since CD200 and HGF have significant clinical relevance, we further demonstrated that, compared with maternal P-MSCs, fetal P-MSCs predominantly express CD200 and HGF prolonged skin allograft survival in vivo and stimulated angiogenesis in vitro, respectively.

MSCs from multiple different tissues have been isolated and characterized. Although bone marrow MSCs remain the most frequently investigated MSC populations, MSCs from different neonatal tissues have recently drawn special attention [26], due in part to their being ethically noncontroversial, easily accessible and MSC-enriched. These MSC origins include amniotic membrane and chorionic membrane [27,28], whole placenta [29], Wharton's jelly [30] and whole umbilical cord [31]. Comparative studies have indicated that different cell sources may have significant impact 


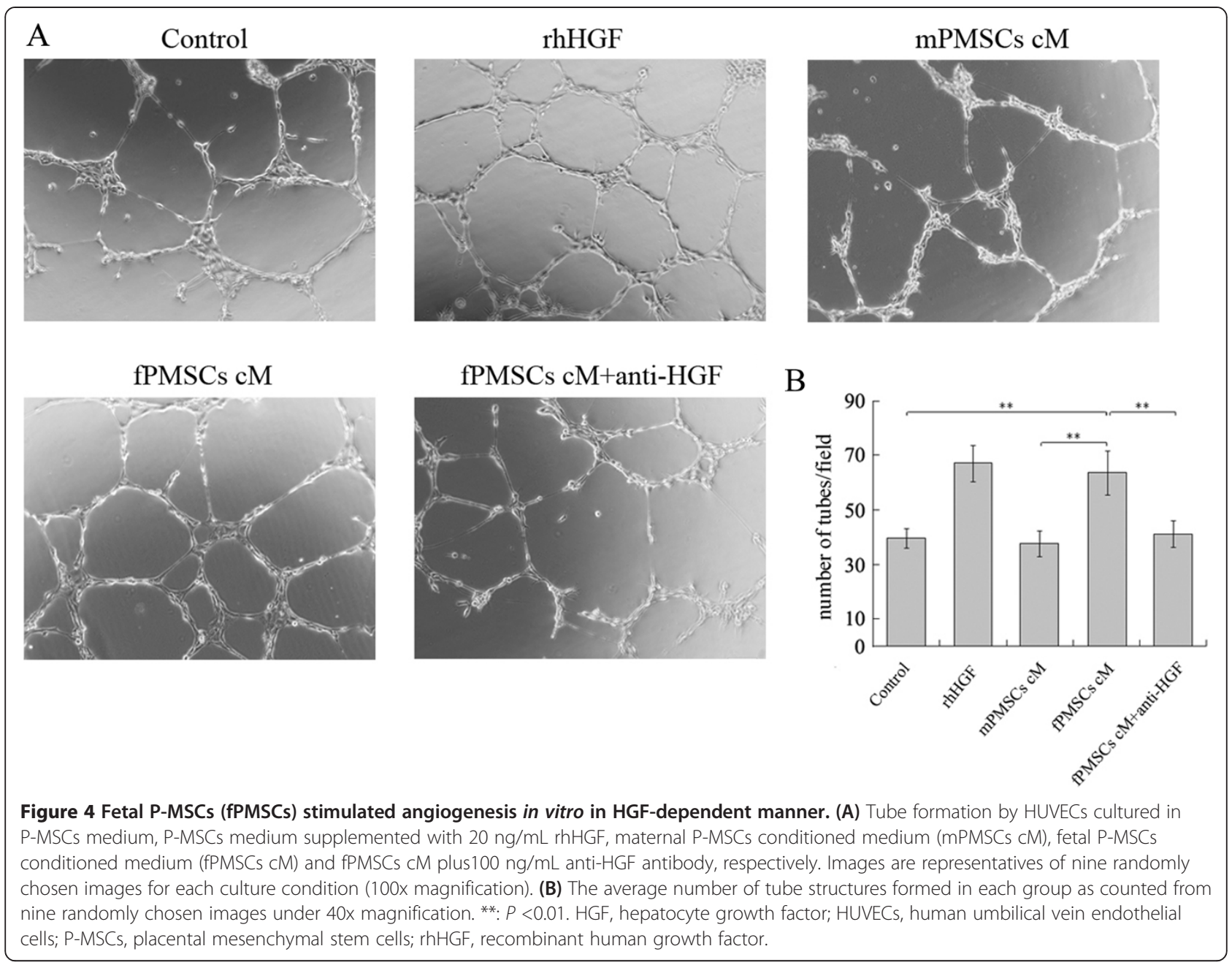

on cell properties. For example, Kern et al. [32] characterized the MSCs from bone marrow, umbilical cord blood and adipose tissue for their stem cell characteristics under identical in vitro conditions, and demonstrated that, although phenotypically similar, these MSC populations exhibited cell source-related heterogeneity in colony frequency, proliferative capability and differentiation potential. Hwang et al. [33] demonstrated that MSCs from placenta, cord blood and bone marrow expressed different cytokine profiles. Gaebel et al. [34] compared MSCs from umbilical cord blood, adipose tissue and bone marrow for their ability to promote cardiac regeneration, and found that MSCs from different sources showed a different healing performance. In our current study, we demonstrated that P-MSCs from the same placenta but different developmental origins have different properties of therapeutic importance, and we further linked these properties to the differential expression of CD200 and HGF by these two MSC populations. These studies altogether support a note that the sources where the MSCs originate may have a significant impact on the therapeutic potential of the cells.
Among the differences between fetal and maternal PMSCs identified in our current study, CD200 and HGF are of specific interest. CD200 is a cell surface glycoprotein mediating an immunosuppressant signal, and has been shown to modulate immune responses by multiple myeloid cell types, especially to dendritic cells and macrophages $[21,22]$. It has been recently reported that cells expressing the CD200 transgene could prolong allograft skin survival [35], and a similar function was demonstrated by using anti-CD200 antibodies [36]. In our present study, we demonstrated that fetal but not maternal P-MSCs expressed CD200 to a significant level, and this expression mediated an anti-rejection function in a mouse skin allograft model. MSCs have been well known for their immunomodulative functions and hence have been tested in multiple clinical settings. Expression of CD200 by MSCs can be thus expected to add clinical benefit when MSCs are applied for immunosuppressive treatments. Pietilä et al. has reported that CD200 was detected in some but not all bone marrow-derived MSC populations and was undetectable in umbilical 


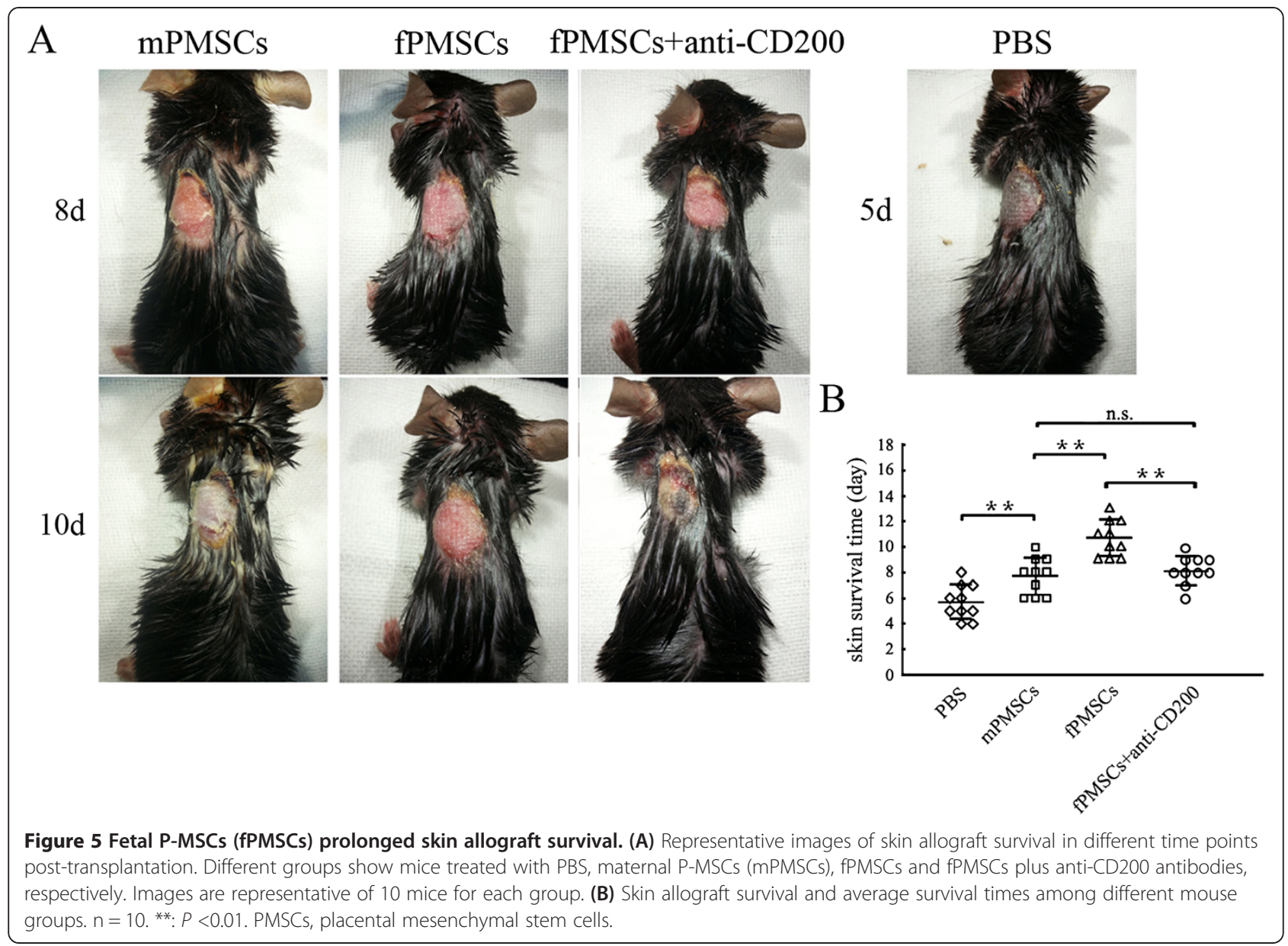

cord blood MSCs [5]. To our knowledge, the present study is the first to report the expression of CD200 in placental MSCs and CD200-mediated immunosuppression by fetal P-MSCs. This finding may provide new insight into the therapeutic potentials of MSCs of neonatal origin. Another finding of particular interest is the preferential expression of HGF by fetal P-MSCs. HGF is a clinically important growth factor that can promote angiogenesis and tissue repair. MSCs have been clinically tested for promoting tissue regeneration and wound healing. Sasaki et al. reported that MSCs contributed to wound repair by transdifferentiation into multiple skin cell types [37]. Wu et al. demonstrated that MSCs enhance wound healing through differentiation and angiogenesis [38]. Chronic wounds and diabetic ulcers, for example, are generally characterized by hyper-inflammation and impaired angiogenesis. While it is possible that MSCs can exert both roles against both dysfunctions, expression of HGF by these cells can be expected to generate enhanced angiogenesis and wound healing. In our study, HGF is highly expressed by fetal P-MSCs but not their partners of maternal origin, indicating that choosing the appropriate MSC type can be expected to generate better clinical outcomes. In this report, we showed evidence that fetal MSC-conditioned medium, similar to recombinant HGF, stimulated angiogenesis in vitro and that the anti-HGF antibody abolished this effect, suggesting the involvement of HGF in MSCmediated angiogenesis. This experiment, however, could not exclude an off-target effect by the antibody and, hence, did not exclusively prove, though it suggested, that the observed angiogenesis is mediated by HGF expression of MSCs. Compared with the maternal P-MSCs that did not express HGF and failed to stimulate angiogenesis, however, the study clearly demonstrated that fetal but not maternal P-MSCS have the ability to enhance angiogenesis. The ultimate demonstration of the mechanisms underlying MSC-mediated angiogenesis is yet to be explored.

\section{Conclusions}

Compared with maternal P-MSCs, fetal P-MSCs express constitutively higher levels of CD200 and HGF, and this difference can mediate different potentials of immunosuppression in vivo and stimulating angiogenesis in vitro, respectively. Taking into consideration that, in the present 
study, all cells and treatments were controlled by the same experimental conditions, and all results were compared between cell population pairs from the same individual donors, the results may suggest that, in general, the sources where the P-MSCs originate may have significant impact on the therapeutic potential of the cells, and, specifically, fetal P-MSCs may be more favorable for applications in cell regeneration, tissue repair and autoimmune disorders where HGF and CD200 may exert a positive effect, and less favorable for applications in immune suppressive cancers where CD200 may mediate breakdown of immunosurveillance and establishment of immune tolerance, or for applications where HGF may enhance tumorsupportive angiogenesis.

\footnotetext{
Abbreviations

BM-MSCs: Bone marrow-derived MSCs; DMEM: Dulbecco's modified Eagles medium; FBS: Fetal bovine serum; FITC: Fluorescein isothiocyanate; fPMSCS: P-MSCs of fetal origin; HGF: Hepatocyte growth factor; HUVECs: Human umbilical vein endothelial cells; IL: Interleukin; INF-y: Interferon gamma; MHC: Major histocompatibility complex; MM: Multiple myeloma; mPMSCs: P-MSCs of maternal origin; MSCs: Mesenchymal stem cells; PE: Phycoerythrin; P-MSCs: Placental MSCs; qRT-PCR: Quantitative reverse transcription-polymerase chain reaction; rhHGF: Recombinant human growth factor.
}

\section{Competing interests}

The authors declare that they have no competing interests.

\section{Authors' contributions}

$J W, Y L$ and $Y Y$ conceived and designed the experiments, and drafted the manuscript. YZZ, YLZ, GH, LW, GZ, TL, TY and QW collected samples, performed experiments, acquired data and drafted the manuscript. YZZ analyzed the data and revised the manuscript. YL interpreted data and critically revised the manuscript. All authors read and approved the final version of the manuscript.

\section{Acknowledgements}

This work was supported in part by a grant of Ningxia Key Science and Technologies R\&D program (2011), Ningxia Natural Science Foundation grant NZ11262 and NZ09133 and Ningxia Medical University Science Program XQ2011025.

\begin{abstract}
Author details
'Institute of Stem Cell Research, General Hospital of Ningxia Medical University, Yinchuan 750004, China. ${ }^{2}$ Key Laboratory of the Ministry of Education for Conservation and Utilization of Special Biological Resources in Western China, College of Life science, Ningxia University, Yinchuan 750021, China. ${ }^{3}$ Key Laboratory of Fertility Preservation and Maintenance, Ministry of Education, Ningxia Medical University, Yinchuan 750004, China. ${ }^{4}$ School of Laboratory Medicine, Ningxia Medical University, Yinchuan 750004, China.
\end{abstract}

Received: 9 June 2013 Revised: 17 December 2013

Accepted: 4 April 2014 Published: 10 April 2014

\section{References}

1. Sekiya I, Larson BL, Smith JR, Pochampally R, Cui JG, Prockop DJ: Expansion of human adult stem cells from bone marrow stroma: conditions that maximize the yields of early progenitors and evaluate their quality. Stem Cells 2002, 20:530-541.

2. Pittenger MF, Mackay AM, Beck SC, Jaiswal RK, Douglas R, Mosca JD, Moorman MA, Simonetti DW, Craig S, Marshak DR: Multilineage potential of adult human mesenchymal stem cells. Science 1999, 284:143-147.

3. De Miguel M, Fuentes-Julián S, Blázquez-Martínez A, Pascual CY, Aller MA, Arias J, Arnalich-Montiel F: Immunosuppressive properties of mesenchymal stem cells: advances and applications. Curr Mol Med 2012, 12:574-591.
4. Majumdar MK, Keane-Moore M, Buyaner D, Hardy WB, Moorman MA, McIntosh KR, Mosca JD: Characterization and functionality of cell surface molecules on human mesenchymal stem cells. J Biomed Sci 2003, 10:228-241.

5. Pietilä M, Lehtonen S, Tuovinen E, Lähteenmäki K, Laitinen S, Leskelä HV, Nätynki A, Pesälä J, Nordström K, Lehenkari P: CD200 positive human mesenchymal stem cells suppress TNF-alpha secretion from CD200 receptor positive macrophage-like cells. PLoS One 2012, 7:e31671.

6. Ben-David D, Livne E, Reznick AZ: The involvement of oxidants and NF-KB in cytokine-induced MMP-9 synthesis by bone marrow-derived osteoprogenitor cells. Inflamm Res 2012, 61:673-688.

7. Neuss S, Becher E, Wöltje M, Tietze L, Jahnen-Dechent W: Functional expression of HGF and HGF receptor/c-met in adult human mesenchymal stem cells suggests a role in cell mobilization, tissue repair, and wound healing. Stem Cells 2004, 22:405-414.

8. Standal T, Abildgaard N, Fagerli UM, Stordal B, Hjertner O, Borset M, Sundan A: HGF inhibits BMP-induced osteoblastogenesis: possible implications for the bone disease of multiple myeloma. Blood 2007, 109:3024-3030.

9. Xu S, Menu E, De Becker A, Van Camp B, Vanderkerken K, Van Riet I: Bone marrow-derived mesenchymal stromal cells are attracted by multiple myeloma cell-produced chemokine CCL25 and favor myeloma cell growth in vitro and in vivo. Stem Cells 2012, 30:266-279.

10. Li X, Ling W, Pennisi A, Wang Y, Khan S, Heidaran M, Pal A, Zhang X, He S, Zeitlin A, Abbot S, Faleck H, Hariri R, Shaughnessy JD Jr, van Rhee F, Nair B, Barlogie B, Epstein J, Yaccoby S: Human placenta-derived adherent cells prevent bone loss, stimulate bone formation, and suppress growth of multiple myeloma in bone. Stem Cells 2011, 29:263-273.

11. Klopp A, Gupta A, Spaeth E, Andreeff M, Marini F 3rd: Concise review: dissecting a discrepancy in the literature: do mesenchymal stem cells support or suppress tumor growth? Stem Cells 2011, 29:11-19.

12. Parolini O, Alviano F, Bergwerf I, Boraschi D, De Bari C, De Waele P, Dominici M, Evangelista M, Falk W, Hennerbichler S, Hess DC, Lanzoni G, Liu B, Marongiu F, McGuckin C, Mohr S, Nolli ML, Ofir R, Ponsaerts P, Romagnoli L, Solomon A, Soncini M, Strom S, Surbek D, Venkatachalam S, Wolbank S, Zeisberger S, Zeitlin A, Zisch A, Borlongan CV: Toward cell therapy using placenta-derived cells: disease mechanisms, cell biology, preclinical studies, and regulatory aspects at the round table. Stem Cells Dev 2010, 19:143-154.

13. Raynaud CM, Maleki M, Lis R, Ahmed B, Al-Azwani I, Malek J, Safadi FF, Rafii A: Comprehensive characterization of mesenchymal stem cells from human placenta and fetal membrane and their response to osteoactivin stimulation. Stem Cells Int 2012, 2012:658356.

14. Lee JM, Jung J, Lee HJ, Jeong SJ, Cho KJ, Hwang SG, Kim GJ: Comparison of immunomodulatory effects of placenta mesenchymal stem cells with bone marrow and adipose mesenchymal stem cells. Int Immunopharmacol 2012, 13:219-224.

15. Abumaree MH, Al Jumah MA, Kalionis B, Jawdat D, Al Khaldi A, Al Talabani AA, Knawy BA: Phenotypic and functional characterization of mesenchymal stem cells from chorionic villi of human term placenta. Stem Cell Rev 2013, 9:16-31.

16. Barlow S, Brooke G, Chatterjee K, Price G, Pelekanos R, Rossetti T, Doody M, Venter D, Pain S, Gilshenan K, Atkinson K: Comparison of human placentaand bone marrow-derived multipotent mesenchymal stem cells. Stem Cell Dev 2008, 17:1095-1107.

17. Jaramillo-Ferrada PA, Wolvetang EJ, Cooper-White JJ: Differential mesengenic potential and expression of stem cell-fate modulators in mesenchymal stromal cells from human-term placenta and bone marrow. I Cell Physiol 2012, 227:3234-3242.

18. Wang L, Yang Y, Zhu Y, Ma X, Liu T, Zhang G, Fan H, Ma L, Jin Y, Yan X, Wei J, Li Y: Characterization of placenta-derived mesenchymal stem cells cultured in autologous human cord blood serum. Mol Med Rep 2012, 6:760-766.

19. In't Anker PS, Scherjon SA, Kleijburg-van der Keur C, de Groot-Swings GM, Claas FH, Fibbe WE, Kanhai HH: Isolation of mesenchymal stem cells of fetal or maternal origin from human placenta. Stem Cells 2004, 22:1338-1345.

20. Roelen DL, van der Mast BJ, In't Anker PS, Kleijburg C, Eikmans M, van Beelen E, de Groot-Swings GM, Fibbe WE, Kanhai HH, Scherjon SA, Claas FH: Differential immunomodulatory effects of fetal versus maternal multipotent stromal cells. Hum Immunol 2009, 70:16-23.

21. Gorczynski R, Chen Z, Kai Y, Lee L, Wong S, Marsden PA: CD200 is a ligand for all members of the CD200R family of immunoregulatory molecules. $\mathrm{J}$ Immunol 2004, 172:7744-7749. 
22. Hoek RM, Ruuls SR, Murphy CA, Wright GJ, Goddard R, Zurawski SM, Blom B, Homola ME, Streit WJ, Brown MH, Barclay AN, Sedgwick JD: Down-regulation of the macrophage lineage through interaction with OX2 (CD200). Science 2000, 290:1768-1771.

23. Matsumoto K, Nakamura T: Hepatocyte growth factor (HGF) as a tissue organizer for organogenesis and regeneration. Biochem Biophys Res Commun 1997, 239:639-644.

24. Benkhoucha M, Santiago-Raber ML, Schneiter G, Chofflon M, Funakoshi H, Nakamura T, Lalive PH: Hepatocyte growth factor inhibits CNS autoimmunity by inducing tolerogenic dendritic cells and CD25 + Foxp3 + regulatory T cells. Proc Natl Acad Sci U S A 2010, 107:6424-6429.

25. Chen D, Ma F, Xu S, Chen F, Rong L, Chi Y, Zhao Q, Lu S, Han Z, Pang A, Han Z: Expression and role of Toll-like receptors on human umbilical cord mesenchymal stromal cells. Cytotherapy 2013, 15:423-433.

26. Hass R, Otte A: Mesenchymal stem cells as all-round supporters in a normal and neoplastic microenvironment. Cell Commun Signal 2012, $10: 26$.

27. Wolbank S, van Griensven M, Grillari-Voglauer R, Peterbauer-Scherb A: Alternative sources of adult stem cells: human amniotic membrane. Adv Biochem Eng Biotechnol 2010, 123:1-27.

28. Soncini M, Vertua E, Gibelli L, Zorzi F, Denegri M, Albertini A, Wengler GS, Parolini O: Isolation and characterization of mesenchymal cells from human fetal membranes. J Tissue Eng Regen Med 2007, 1:296-305.

29. Brooke $\mathrm{G}$, Tong $\mathrm{H}$, Levesque JP, Atkinso K: Molecular trafficking mechanisms of multipotent mesenchymal stem cells derived from human bone marrow and placenta. Stem Cells Dev 2008, 17:929-940

30. Wang HS, Hung SC, Peng ST, Huang CC, Wei HM, Guo YJ, Fu YS, Lai MC, Chen CC: Mesenchymal stem cells in the Wharton's jelly of the human umbilical cord. Stem Cells 2004, 22:1330-1337.

31. Majore I, Moretti P, Stahl F, Hass R, Kasper C: Growth and differentiation properties of mesenchymal stromal cell populations derived from whole human umbilical cord. Stem Cell Rev 2011, 7:17-31.

32. Kern S, Eichler H, Stoeve J, Klüter H, Bieback K: Comparative analysis of mesenchymal stem cells from bone marrow, umbilical cord blood, or adipose tissue. Stem Cells 2006, 24:1294-1301.

33. Hwang JH, Shim SS, Seok OS, Lee HY, Woo SK, Kim BH, Song HR, Lee JK, Park YK: Comparison of cytokine expression in mesenchymal stem cells from human placenta, cord blood, and bone marrow. J Korean Med Sci 2009, 24:547-554.

34. Gaebel R, Furlani D, Sorg H, Polchow B, Frank J, Bieback K, Wang W, Klopsch C, Ong LL, Li W, Ma N, Steinhoff G: Cell origin of human mesenchymal stem cells determines a different healing performance in cardiac regeneration. PLoS One 2011, 6:e15652.

35. Gorczynski RM, Chen Z, Khatri I, Yu K: Graft-infiltrating cells expressing a CD200 transgene prolong allogeneic skingraft survival in association with local increases in Foxp3 (+) Treg and mast cells. Transpl Immunol 2011, 25:187-193.

36. Rygiel TP, Luijk B, Meyaard L: Use of an anti-CD200 antibody for prolonging the survival of allografts: a patent evaluation of WO2012106634A1. Expert Opin Ther Pat 2013, 23:389-392.

37. Sasaki M, Abe R, Fujita Y, Ando S, Inokuma D, Shimizu H: Mesenchymal stem cells are recruited into wounded skin and contribute to wound repair by transdifferentiation into multiple skin cell type. J Immunol 2008, 180:2581-2587.

38. Wu Y, Chen L, Scott PG, Tredget EE: Mesenchymal stem cells enhance wound healing through differentiation and angiogenesis. Stem Cells 2007, $25: 2648-2659$

doi:10.1186/scrt436

Cite this article as: Zhu et al:: Placental mesenchymal stem cells of fetal and maternal origins demonstrate different therapeutic potentials. Stem Cell Research \& Therapy 2014 5:48.

\section{Submit your next manuscript to BioMed Central and take full advantage of:}

- Convenient online submission

- Thorough peer review

- No space constraints or color figure charges

- Immediate publication on acceptance

- Inclusion in PubMed, CAS, Scopus and Google Scholar

- Research which is freely available for redistribution

Submit your manuscript at www.biomedcentral.com/submit 